\title{
Using Real-Time Chemical Plume Models In Virtual Training Systems
}

\author{
Adam Moses, Keith Obenschain, Dr. Jay Boris, Dr. Gopal Patnaik \\ Laboratories for Computational Physics \& Fluid Dynamics \\ US Naval Research Laboratory \\ Washington, DC, USA \\ adam.moses@nrl.navy.mil
}

\begin{abstract}
This paper describes the design, implementation, and use of integrated chemical plume-models in virtual training systems. The US Naval Research Laboratory has linked its CTAnalyst ${ }^{\circledR}$ software, a high-fidelity real-time plume modeling tool, with VBS2, a widely-used virtual gaming and training program, to produce new training capabilities that were previously unavailable. This work benefits two different but overlapping training scenarios: 1) tactical training for large-scale chemical gas attacks with a specific focus on crowd management, and 2) handler-focused training for users interested in working with IED-detecting dogs. The use of accurate, faster-than-real-time plume modeling enhances the virtual training systems to provide broader realistic support to the simulation and training communities.
\end{abstract}

Keywords-plume modeling; hazardous materials; chemical agents; contaminants; dog handler; improvised explosive device detection; virtual training; serious gaming; simulation; virtual battle space

\section{BACKGROUND}

Modern virtual training systems are adopting gaming platforms as a means to prepare military personnel and civilian first responders [1]. Gaming platforms offer a realistic, threedimensional, virtual world that immerses users in relevant, realistic scenarios to create more robust and meaningful learning experiences. These gaming platforms allow task specific scenario-building, which includes complex interaction with other actors and with items, weapons, and vehicles in environments specific to a training goal. Gaming platforms also offer a greater variety of scenario-development tools, after-action metrics, and customization than can be available in real-world exercises.

As gaming platform adaptation for virtual training progresses, in-game capabilities continue to improve, bringing the simulations closer and closer to real life. There is a distinct lack of reliable, faster-than-real-time plume modeling in popular modern gaming platforms. Current platforms at most offer gas cloud effects that are pre-scripted in some way, and even these do not incorporate proper 3-D modeling. Further the treatment of health effects is primitive. A good plume model needs to capture not only the propagation effects caused by the in-game geometry, but must also properly predict agent concentrations and contamination effects.
To this end the US Naval Research Laboratory (NRL) in Washington, DC has identified a major area where significant improvements are possible, specifically the use of accurate chemical plume models to enhance training. NRL had been developing the high fidelity, real-time, geometrically-accurate plume modeling tool Contaminant Transport Analyst (CTAnalyst $\left.{ }^{\circledR}\right)$ for over ten years. As we will describe, integrating CT-Analyst into the widely used gaming platform Virtual BattleSpace 2 (VBS2) allowed for improvements that enable new and relevant training programs. For this project two different tasks were undertaken: tactical training for chemical gas attacks and dog handler training for teams attempting to detect improvised explosive devices (IED).

\section{CT-ANALYST}

Older plume modelling applications suffer from being too difficult to use and they require significant time for scenario predictions [2]. NRL tackled this problem in the early 1990s by developing CT-Analyst. CT-Analyst is an urban focused, real-time, high-fidelity plume model that produces results in a few milliseconds, as opposed to minutes or even hours that other high-fidelity plume models require [3].

\section{A. Pre-Computed Plume Models}

CT-Analyst achieves its large speed advantage by precomputing plume results in the form of Nomograf Tables ${ }^{\mathrm{TM}}$. Nomografs are a compressed representation derived directly from much larger time-dependent, compute-intensive 3-D computational fluid dynamics (CFD) calculations [4]. These CFD calculations, which are precomputed for each given geographical region, predict wind fields that capture how an airborne plume travels over complex city geometry with detailed buildings and terrain. Once determined, these wind fields are distilled down into the Nomograf Tables, enabling quick lookups as opposed to spawning a complete new calculation every time an input parameter is changed.

Nomograf Tables offer not only a massive speed advantage by reducing what is ordinarily a demanding 3-D calculation into something closer to a database lookup, but also greatly simplify deployments because of the lessened computational requirements. The Nomograf Tables used in CT-Analyst are at most a few hundred megabytes in size so a typical Windows or Mac installation can run on any platform from a high-end workstation to a bare-essentials mobile laptop. 


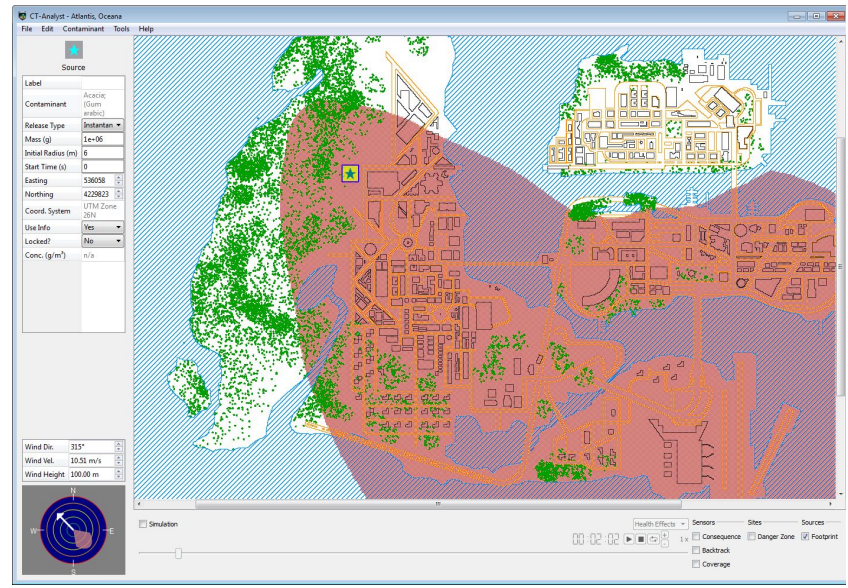

Fig. 1. The application window of CT-Analyst where the pink shaded area shows the harzard area, also called the footprint. In this case the footprint is rendered over the ficticous region of "Atlantis".

From a deployment perspective CT-Analyst is designed to be simple to use and require minimal training. As Fig. 1 shows, the graphical user interface (GUI) is simple, reducing the complexity of the terms and commands involved to only what is absolutely required by an operator of CT-Analyst.

The pre-computed nature of the Nomograf Tables might suggest that CT-Analyst is only suited for training purposes, however this is not the case. With sufficient lead-time to acquire the necessary 3-D geometry of a region and run this information through the Nomograf Table production process, which varies between a week to several months depending on input data quality, the end result produces a Nomograf Table suitable for operational purposes.

\section{B. Integration Efforts}

CT-Analyst's unique speed advantage over other plume models also makes it a powerful tool that can provide airborne plume model results to other applications. CT-Analyst was built to be used as a data engine by other applications, via a programming interface as well as through other methods, and thus can be integrated easily into programs like military simulators, Geographic Information System packages, and sensor aggregation systems.

CT-Analyst capability has been used to improve the rudimentary plume model in the US Army's One SemiAutomated Forces (OneSAF) entity-based simulation software. In this OneSAF installation, generic oval-shaped smoke clouds were replaced with CT-Analyst terrain-aware modeled results, enabling accurate line-of-sight calculations to be made based on the calculated plume densities. The region modeled by CTAnalyst was from the identical terrain and building geometries used by OneSAF.

CT-Analyst has also been used as a chemical-plume overlay for situational awareness systems from companies such as Safe Environment Engineering, Defense Group Incorporated, and Priority 5 Incorporated. The fidelity and simplicity of CT-Analyst has also led to its operational deployment in support of three inaugurations and other major events such as the Super Bowl. A German-language version of
CT-Analyst is currently in use 24/7 in Hamburg Germany for port and city security.

\section{ViRTUAL BATtLeSPACE 2}

VBS2 is a fully interactive, three-dimensional, PC-based virtual environment suitable for military and civilian training and scenario development. Developed by Bohemia Interactive, VBS2 supports rapid, real-world terrain development and operates along with a number of the top simulation standards. The Army, Marines, and civilian law enforcement use VBS2 extensively, so NRL chose VBS2 as the gaming platform for the two projects described here [5].

VBS2 features a vast built-in library of in-game assets ranging from avatar models like soldiers, civilians, and insurgents, to a wide assortment of weapons, vehicles, structures, and regional terrains. This pre-existing infrastructure means not having to find and re-introduce many important training elements, another important factor in the selection of VBS2 for this development. Fig. 2 illustrates this by showcasing a scene in VBS2 composed entirely of preexisting assets.

Beyond the industry support and infrastructure assets, VBS2 offers another key feature that made it an attractive gaming platform to develop with. The VBS2 Application Scripting Interface (ASI) is a programming interface that allows developers to interact with objects inside the gaming platform programmatically to enable more complex scenarios and effects than can be achieved by VBS2 alone [6].

\section{Chemical Symptom TRAINER}

The goal of the chemical symptom trainer was to simulate the effects of an airborne toxic chemical attack on a crowd of people. Scenarios imagined for this goal were terrorist attacks against gatherings of people such as a political rally or concert. The player must first learn to detect and understand the effects of the chemical agent and then take appropriate steps to respond.

This goal has two key requirements. The first is to visually render the chemical plume cloud as it moves realistically through the virtual environment, taking into account the

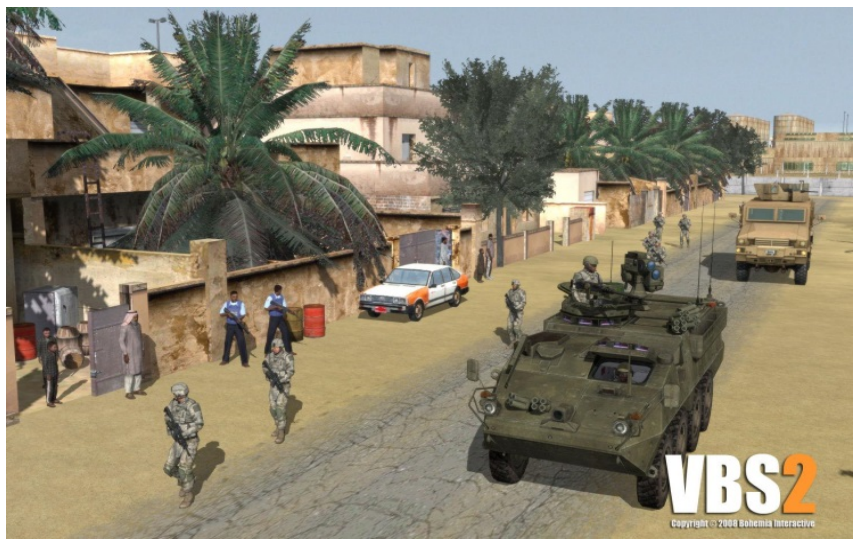

Fig. 2. A military convoy scene rendered in VBS2, complete with detailed terrain, buildings, vegetation as well as the interactive elements of the avatars and vehicles. 
building geometries and terrain as well as the wind speed and direction. An example is shown in Fig. 3 below. For some applications the visibility of the cloud would need to be turned off as training often deals with odorless, invisible gases. The second requirement is that the avatars, both computercontrolled and human, should experience health effects based on dose calculations using the changing positions of the avatars and the chemical plume evolution over time. Once the dose is known the appropriate effects on the avatar can then be rendered by animation in the on-screen display.

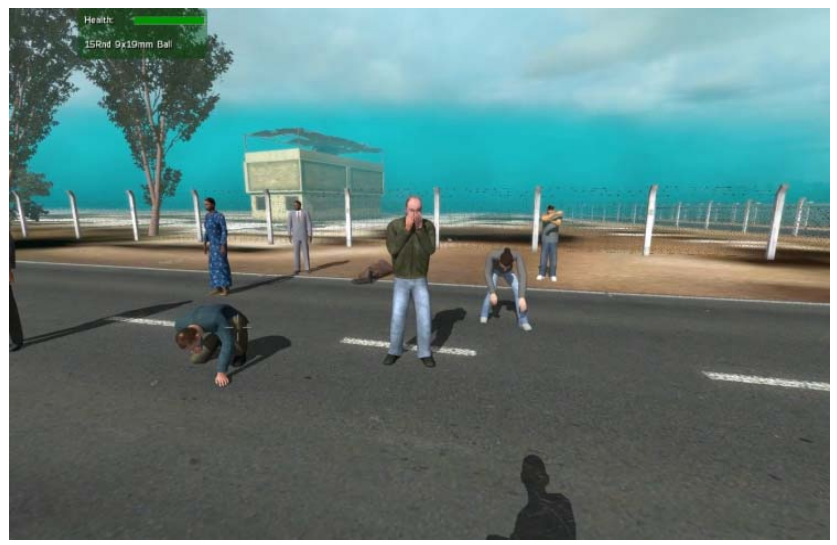

Fig. 3. A VBS2 screenshot demonstrating the CT-Analyst plume model applied inside the virtual environment. The plume cloud is shown as the visible blue-green cloud while health effects are demonstrated by the coughing and kneeling over by the in-game avatrs.

\section{A. Plume Cloud Visualisation}

Out of the box, VBS2 featured only basic plume visualization without any chemical effects. The visuals were created using in-game programmable particle generation stations. These stations produced individual "puffs" seen by the user, with each puff behaving according to the initial input parameters of the individual particle generation station. Parameters for these stations included speed, size, color, and the rate at which puffs were produced. Once a station creates a puff it can no longer be modified. Thus the appearance of the puffs can be controlled only by what has been pre-scripted.

Because CT-Analyst can provide real-time terrain and geometry-aware plume results that are modifiable to accommodate scenario changes such different wind directions or addition of new user-generated sources, the inclusion of CTAnalyst results brings a vast improvement in operations available within VBS2. We describe the incorporation of CTAnalyst into VBS2 below.

As contaminant sources are added to a scenario in VBS2 using the ASI, their locations are sent to the CT-Analyst application running in the background. CT-Analyst uses the source locations to produce a rasterized concentration plume (based on the current in-game time), that is to say a series of fixed points whose locations are matched between VBS2 and CT-Analyst. Once these locations and their corresponding contaminant concentration values are known the interface generates the necessary particle (puff) generation stations inside of VBS2 at the appropriate locations. These stations are programmed to match the strength, spatial distributions, and speed of the CT-Analyst plume in order to produce corresponding results within the VBS2 rendering.

At defined intervals, this operation is repeated with the previous particle generation stations eliminated and new ones added based on the scenario changes since the last update. This loop runs continuously so that the visual results reflect the latest transport and dispersion of contaminants and match the specific source and environmental characteristics as well. This method also ensures the visual plume will appear to spread continuously even though total geometric area of the plume may be discontinuous between update intervals.

\section{B. Health Effects}

Combining the VBS2 particle generation system, the ASI, and running CT-Analyst as a plume model in the background, NRL was able achieve a very robust plume visualization system. This system visually renders plumes in a way previously unavailable, with plume transport based on the actual in-game three-dimensional terrain, building geometry, and scenario evolution, and with the color, density, and velocity of the plume all specified by the physics-based simulation of the plume.

The VBS2 ASI makes it possible to track the location of all the avatars in the game. Their instantaneous location is used to extract the concentration level they would experience in the time-dependent chemical cloud generated by CT-Analyst. This local concentration is then used to calculate a total accumulated dose over time for each of the moving avatars. By comparing the accumulated dose to the dosage needed for the onset of a specific toxicological heath effect, a determination of the health of the avatar can be made. This is used to trigger an animation of a specific resulting behavior, coughing to the side, rubbing eyes, and rubbing of the neck. Once the dose is determined to have reached lethal levels, the avatar can be rendered dead inside the VBS2 environment. Fig. 3 is a screen capture from the chemical trainer system. The modeled plume cloud is shown as the blue-green gas in the background, while the physical reactions exhibited by the in-game avatars show the health effects of the toxic chemical plume.

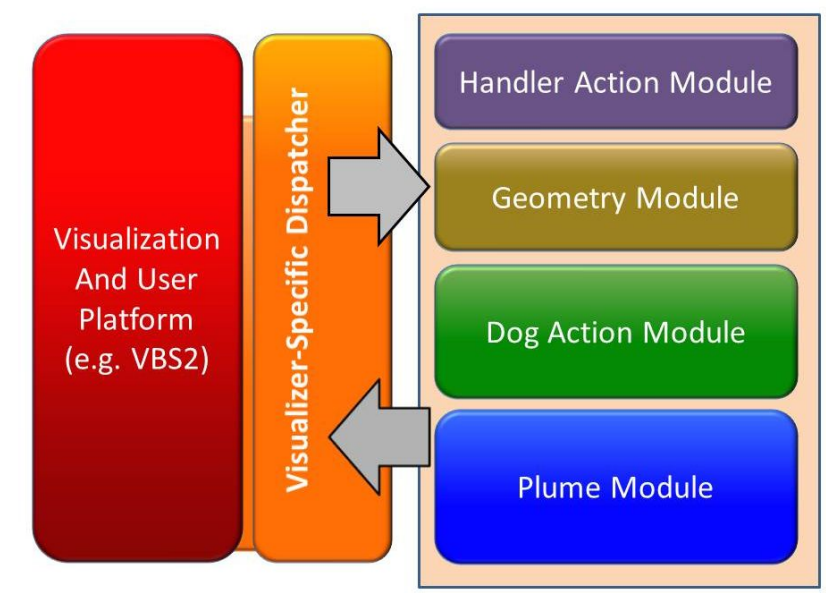

Fig. 4. A visual breakdown showing the various modules that comprised the ROVER system. 


\section{IED DOG HANDLER TRAINER}

The goal of the dog handler trainer project was to provide a virtual training solution for handlers who are separated from their military improvised explosive device (IED) detection dogs for long durations between their training with the dog and the time they are re-united with their dog in the field [7]. In many instances this delay can be months, during which the handler's skills diminish and necessitate significant re-training when they are paired with the dog again.

To help solve this problem, NRL developed a virtual IED detection dog handler training system, called ROVER. ROVER is a first of its kind simulator that combines a model of dog behavior with a realistic plume model of scent vapors from a buried IED and with the capability to interact with appropriate real-life hand gestures. Using VBS2, NRL was able to implement a training environment that allows for the dog handler to interact with a virtual dog that responds to both the handler's own physical gesture and whistle commands while tracking the IED vapor trail upwind to uncover a buried IED.

\section{A. Modular Design}

ROVER was designed as a system of modules each contributing a unique capability to the larger system. The primary reason to structure ROVER this way was so that if any part of the system was improved or needed modification, i.e. using software other than VBS2 for visualization, this could be accomplished without needing to replace or modify components throughout the entire system.

This modular design divided the work into several principal parts, as shown schematically in Fig. 4. These include handler actions, dog actions, geometry-based decisions, and plume modeling information. The combination of the inputs and outputs from these modules was then synced with the VBS2 environment for rendering the dog, handler, and other objects in the scene. The VBS2 geometry had to be reproduced faithfully in the CT-Analyst CFD computations and in the CTAnalyst plume rendition to ensure that the physics-based modeling was performed with the identical geometry.

\section{B. Physical Gesture Handling}

The fidelity of this ROVER training simulator was greatly enhanced using an off-the-shelf XBox Kinect camera system, commonly used to play interactive motion and dancing games or to control a home entertainment system, as part of the handler action module. The Kinect was used to monitor the dog handler and watch for physical gestures that signal required actions to the dog such as "go left" or "sit at my right". Using these physical gestures, the handler interacts with the virtual dog just as he or she would interact with a trained IED detection dog in the real world.

Conveniently, the Kinect has a well-developed programming interface that automatically creates a "skeleton" representative of the handler who is standing in front of the camera [8]. This skeleton representation can then be interrogated for markers like body positions, elbow angles, and so on, which are used to determine if the handler is issuing a gestured signal to the dog. This gesture is parsed and interpreted by the handler action module and the result sent to the dog action module for further decision on if, how and when to act on it on the gesture command.

\section{Dog Action (Behavior) Model}

The dog action module was responsible for determining the moment to moment behavior of the dog, i.e. following the scent of the buried IED while responding to handler signal gestures, and for determining which path to take towards an unseen target location in a complex geometry. Because this dog action module is so central to ROVER, it directly interfaced with all other modules to determine the evolving dog avatar behavior. This connectivity is shown schematically in Fig. 4.

Using data from the plume module, the dog action module determines what level of scent (if any) was there to be detected and determine the direction from which the odor was emanating. The dog would proceed in that direction, unless commanded otherwise by the handler. Eventually the dog would arrive where it "believed" the IED was most likely buried and exhibit the appropriate behavior (Fig. 4 left). Watching videos of working dogs and experimentation with
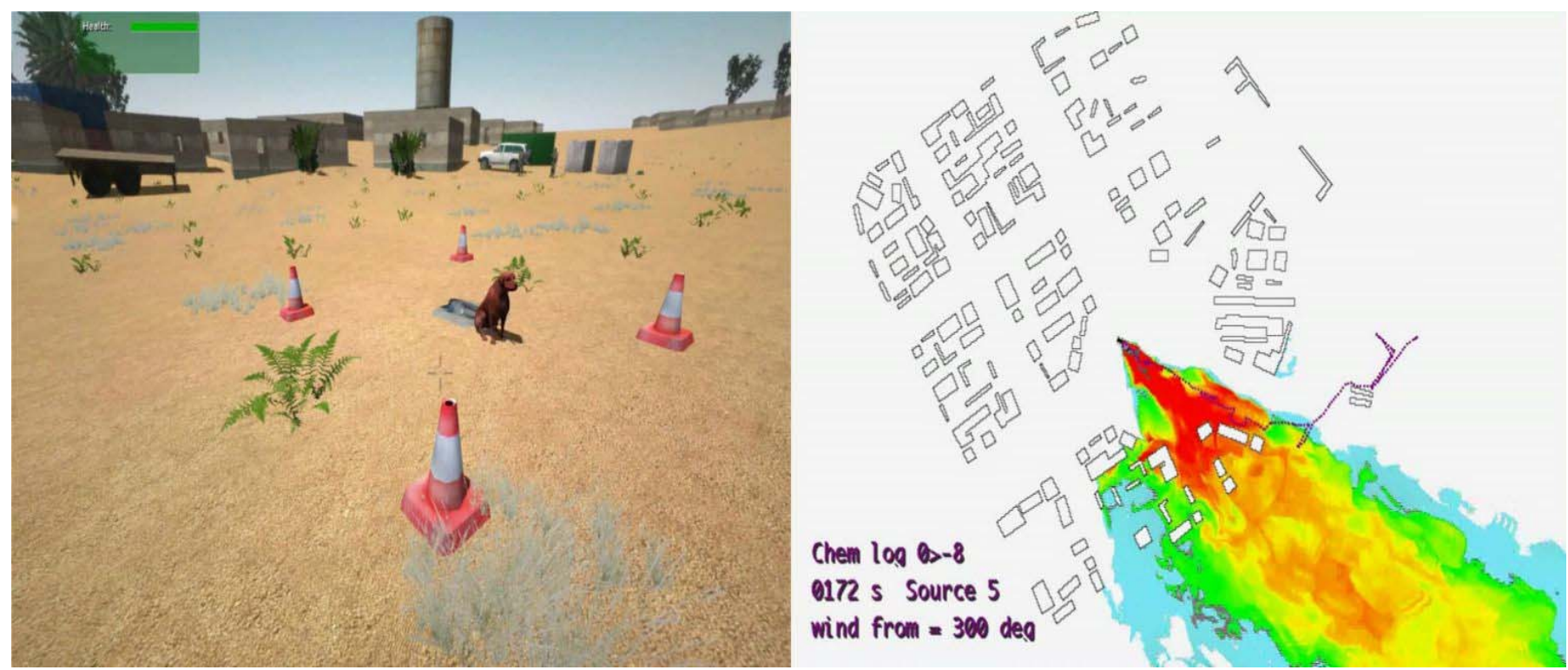

Fig. 5. On the left a screenshot of the ROVER training system, where the dog has located the buried IED. On the right is the plume model dispersion field for trace scents being released into the environment by the IED. The purple line illustrates the path the dog took to find the IED, also accounting for the handler gestures which sent the dog in certain directions as well as at least once calling for the dog to return to the handler. 
the ROVER system showed that naturally-occurring fluctuations in the unseen IED scent plume played a very big part in the complexity of the dog's behavior and had to be accounted for in the final system.

The geometry module shown in Fig. 4 was used to determine the dog's route around objects and how to return to the handler quickly. Finally interaction with the handler action module also provides information not just about the physical signal gestures and thus the appropriate action to be taken but also about the position of the handler, whistle (sound) commands, and other pertinent details.

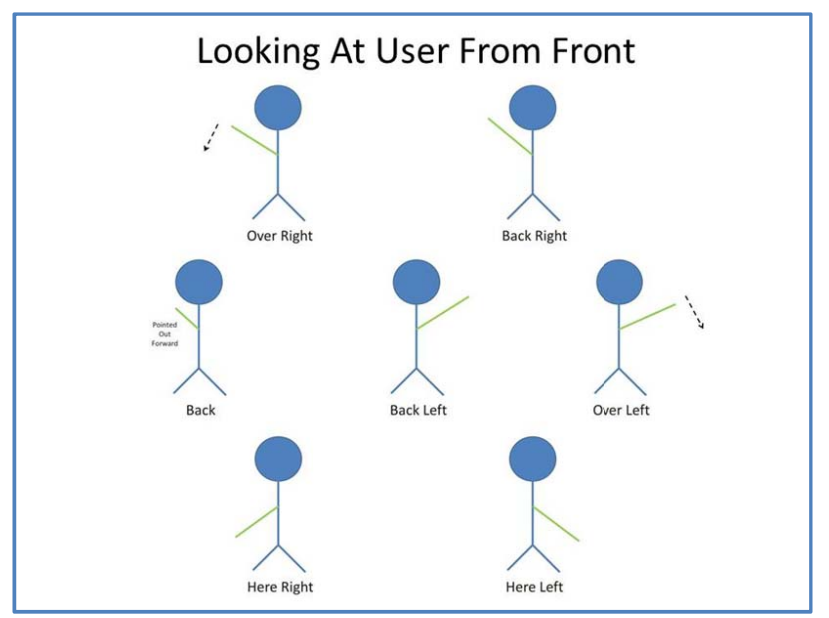

Fig. 6. A guide to the gestures used by dog handlers to signal movement commands to their dogs. These gestures were based on the identical movements used by actual dog handlers in the field.

\section{Example Scenario}

The goal of this example scenario is to find a buried IED somewhere in the virtual VBS2 landscape. Physically, the handler trainee stands in front of a large monitor displaying VBS2 and in front of the properly oriented Xbox Kinect. The trainee also holds an Xbox game controller that they use to move in and look around the VBS2 virtual world. The game controller also offered the use of a button to sound a virtual "whistle" to get the dog's attention, as in the field.

Starting with the dog facing him in the virtual world, the handler would then gesture a command like "go back", which signals the dog to begin moving in the direction the handler is facing. As the dog moves, the handler follows it using the game controller to move to effect motion within the training simulation. If the dog is moving in an undesired direction, the handler can press a button to issue a whistle, making the dog stop, and then issue a new gesture like "go left" or "go right". The available gestures are indicated schematically in Fig. 6.

As the dog moves it will eventually encounter the odor plume of the buried IED, and then turns upwind to find the source location. Along the way the handler could still issue new commands to alter the course of dog if the handler felt he or she had other indicators of the location of the buried IED. Once the dog has located the origin of the odor plume, it stops and sits at that location.

Fig. 5 illustrates a ROVER scenario with a dog having found the buried IED on left of the image, and on the right is a scenario monitoring utility showing an overhead 2-dimensonal map of the virtual area. The purple line indicates the route traveled by the dog, including some sharp turns as a result of a new handler command or discovering the odor plume.

\section{CONCLUSIONS}

The chemical symptom trainer and ROVER embody significant developments for virtual training as both use detailed chemical plume models in an entirely new way. By using CT-Analyst, both of these projects received the benefits of a high-fidelity chemical plume model able to respond to changes made at any time during a scenario without requiring extensive recalculations of a result. For the chemical symptom trainer this means providing the in-game visualization of the time-dependent plume as well as calculating realistic and evolving health effects experienced by the avatars in VBS2. For ROVER this provided the accurate modeling of the downwind flow of the scent plume from a hidden IED, giving a better chance at capturing the dog behavior a handler would be expected to experience in training and on deployment.

\section{A. Project Deployments}

The chemical symptom trainer was demonstrated to a number of military and civilian agencies, both domestic and foreign, in an attempt to further inform training specialists and decision makers on the advanced plume modeling capability available to them. The chemical symptom trainer has been used as part of an integrated virtual training system by government agencies.

ROVER was completed as a beta-level demonstration project to the Office of Naval Research as part of their Science and Technology directorate. The principal deployment for ROVER has been the delivery of a working beta test system to a dog handler training facility in North Carolina, and is currently undergoing feedback and assessment. ROVER has also been showcased more largely at canine training seminars as a novel way to provide training en masse without having to use actual dogs.

\section{B. Future Work}

The goal of these projects, to further extend the capabilities of virtual training systems with fast and accurate contaminant plume models, will lead to other virtual training applications. The chemical symptom trainer is intended to be a first step towards a more fully comprehensive training suite for chemical attack scenarios. The training environment could be improved to handle more dynamic assets like hazardous material suits, dispersal agents, and more complex computer-controlled behaviors exhibited by affected avatars.

ROVER also has potential as a virtual training system for drug-enforcement dog handler training, which would be a logical next step as it involves the same kind of plume modeling requirements and the sound-based and physical gesturing that the IED detection scenario required.

\section{ACKNOWLEDGMENT}

The authors would like to thank the Naval Research Laboratory for the continual support of CT-Analyst and its 
associated projects in the field of plume modeling. The authors thank Lisa Albuquerque and Mike Hoglund for their leadership roles in ROVER and the support for it found in the Office of Naval Research. Also deserving recognition for their critical contributions to the visualization, Kinect and animation components of ROVER are Mark Livingston and Zhuming Ai, also of the Naval Research Laboratory. The authors also thank Bohemia Interactive and Gryphon Scientific.

\section{REFERENCES}

[1] Maj. P. Zamora, "Virtual training puts the 'real' in realistic environment". Published March 2013 Available: http://www.army.mil/article/97582/

[2] J. Boris, "The threat of chemical and biological terrorism: preparing a response," Computing in Science \& Engineering, vol.4, no.2, pp.22,32, March/April 2002
[3] J. Boris, K. Obenschain, G. Patnaik, T. Young, "CT-ANALYST TM , Fast And Accurate CBR Emergency Assessment", Proceedings of the 2nd International Conference on Battle Management, November 2002

[4] J. Boris, "Dust in the Wind: Challenges for Urban Aerodynamics", AIAA Paper 2005-5393, pp. 1-63, June 2005

[5] A. Moses, J. Boris, K. Obenschain, Using CT-Analyst as an integrated tool for CBR analysis, SPIE Proceedings, vol 6201 , no , pp.1-11, May 2006

[6] "Bohemia Interactive Simulations Releases VBS2 v.20", Published November 2011, Available: https://bisimulations.com/content/mon11282011-1036/bisim-releases-vbs2-v20

[7] K. Crawford, "Marine's Best Friend Shows Explosive-Detecting Capabilities", Published Janurary 2012, Available: http://www.onr.navy.mil/en/Media-Center/Press-Releases/2012/IEDExplosive-Device-Detect-Dog.aspx

[8] J. Kennedy, "Skeltrack: Tracking the Human Skeleton With Kinect". Published March 2012, Available: http://www.techhive.com/article/252503/skeltrack_tracking_the_human skeleton_with_kinect.html 